Article

\title{
The Potential Transformation Mechanisms of the Marker Components of Schizonepetae Spica and Its Charred Product
}

\author{
Xindan Liu ${ }^{\mathbb{D}}$, Ying Zhang *, Menghua Wu, Zhiguo Ma and Hui Cao * \\ Research Center for Traditional Chinese Medicine of Lingnan (Southern China), Jinan University, \\ Guangzhou 510632, China; liuxindan66@stu2018.jnu.edu.cn (X.L.); zyfxwmh@163.com (M.W.); \\ mzg79@hotmail.com (Z.M.) \\ * Correspondence: zhangying@jnu.edu.cn (Y.Z.); huicao@stu2015.jnu.edu.cn (H.C.) \\ Academic Editor: Derek J. McPhee \\ Received: 15 July 2020; Accepted: 17 August 2020; Published: 17 August 2020
}

\begin{abstract}
Schizonepetae Spica (SS) is commonly used for treating colds, fevers, bloody stool and metrorrhagia in China. To treat colds and fevers, traditional Chinese medicine doctors often use raw SS, while to treat bloody stool and metrorrhagia, they usually use Schizonepetae Spica Carbonisata (SSC; raw SS processed by stir-frying until carbonization). However, there have been limited investigations designed to uncover the mechanism of stir-fry processing. In the present study, a method combining gas chromatography-mass spectrometry (GC-MS) and high-performance liquid chromatography (HPLC) was developed for the comprehensive analysis of the chemical profiles of SS and SSC samples. Principal component analysis of the GC-MS data demonstrated that there were 16 significant differences in volatile compounds between the SS and SSC samples. The simultaneous quantification of six nonvolatile compounds was also established based on HPLC, and remarkable differences were found between the two products. These changes were probably responsible for the various pharmacological effects of SS and SSC as well as the observed hepatotoxicity. Finally, the mechanisms could be rationalized by deducing possible reactions involved in the transformation of these marker components. This work reports a new strategy to reveal the chemical transformation of SS during stir-fry processing.
\end{abstract}

Keywords: Chinese medicinal material; Schizonepetae Spica; Schizonepetae Spica Carbonisata; stir-frying; marker component; transformation mechanism

\section{Introduction}

Chinese medicinal materials (CMMs) often have to be processed by using physical or chemical methods before prescription or clinical usage. The aims of processing are to alter the clinical efficacy and/or reduce the toxicity of the CMMs [1]. The classic Chinese medicine literature Lei Gong Pao Zhi Lun (Lei Gong Processing Handbook, 500 A.D.) emphasized the importance of processing for the first time as a pharmaceutical technique to fulfill the different requirements of therapy [2]. Modern research has indicated that the processing of CMMs has a large influence on the quality and quantity of chemicals in medicinal materials, which consequently affects the bioactivities, pharmacokinetics, and/or safety of the medicinal materials [3-6].

Schizonepetae Spica (SS) is the dried spike of Schizonepeta tenuifolia Briq. (Chinese Pharmacopoeia, 2015 edition) [7]. It was first recorded in Shen Nong Ben Cao Jing (Shen Nong's herbal classic), a book written 2000 years ago [8]. The herb is commonly used in traditional Chinese medicine (TCM) prescriptions to treat colds, fevers, bloody stool and metrorrhagia [9]. To treat colds and fevers, TCM practitioners often prescribe raw SS, while to treat bloody stool and metrorrhagia, they 
usually use Schizonepetae Spica Carbonisata (SSC; raw SS processed by stir-frying until carbonization). Pharmacological analyses have shown that SS had three main biological and pharmaceutical properties, including anti-inflammatory [10], antiviral [11,12] and hemostatic activity [13-15]. Chemical studies revealed that SS contains volatile oils, flavonoids, organic acids, etc. [16-18]. The essential oils accumulated by SS are recognized as the major constituents responsible for its anti-inflammatory and antiviral effects $[10,19]$. One of the major pharmacological volatile components in SS is pulegone, which is also used as a biomarker for SS in the Chinese pharmacopoeia (2015 edition) [7]. However, evidence has demonstrated that pulegone can cause severe hepatotoxicity [20]. Previous reports have shown that changes in the quantity and quality of the components in the volatile oils of Schizonepetae Herba (SH, the aerial part of S. tenuifolia Briq.) and its charred product Schizonepetae Herba Carbonisata (SHC; raw SH processed by stir-frying until carbonization) have been observed: nine new components were formed and eight components disappeared in the volatile oils after processing while the contents of seven original constituents decreased and the concentration of nine other constituents increased [21]. The changes in the contents of some constituents between the raw drugs and processed products are mostly due to the transformation of chemical structures and/or volatilization of volatile compounds during processing. However, the possible mechanism involved in the transformation of marker compounds induced by processing by stir-frying SS has not been fully elucidated. The influence of processing on SS is not only due to the change in volatile oils, and further study on the change in other components is needed.

In an attempt to uncover the potential mechanism of stir-fry processing on SS, a combination of gas chromatography-mass spectrometry (GC-MS) and high-performance liquid chromatographydiode-array detector (HPLC-DAD) was carried out, thus obtaining an overall characterization for both volatile and nonvolatile compounds. The information obtained from GC-MS was then analyzed by chromatographic fingerprinting to compare the chemical profiles of SS and SSC. Statistical analyses of principal component analysis (PCA) from GC-MS and Student's two-tailed $t$-tests from HPLC were employed to find marker compounds to differentiate SS from SSC scientifically and reliably. The processing mechanism could be rationalized by deducing possible reactions involved in the transformation of these marker compounds.

\section{Results and Discussion}

\subsection{Comparison of SS and SSC Volatile Compounds}

\subsubsection{GC-MS Fingerprint of the Essential Oils from SS and SSC}

The essential oils from the SS and SSC samples were extracted by hydrodistillation. The essential oil yield of SS markedly decreased after stir-fry processing, and the essential oil yield from SSC was too low to be detected. It has been reported that stir-frying can dramatically reduce the contents of volatile components from SS through heat volatilization [22]. Nonetheless, the essential oil can still be detected after stir-frying, which supports the theory of CMM processing "raw medicinal herbs processed by stir-frying until carbonized to avoid scorching" [23,24]. All samples were analyzed by GC-MS, and the chromatograms are shown in Figure 1. The correlation coefficient of similarity between each chromatographic profile of SS, SSC and their reference chromatogram, the representative standard fingerprint/chromatogram for a group of chromatograms, was calculated (Table 1). The correlation coefficients from each chromatogram for 11 SS batches were found to be 0.969-0.997, which was in agreement with previous studies [25,26], while those from the 11 batches of SSC were $0.955-0.995$ (Table 1). These results demonstrated that the chromatographic fingerprints of SS and SSC were consistent to some extent despite their slightly different chemical compositions, which indicated that the developed processing method for investigating the specific variations between SS and SSC was satisfactory. After stir-fry processing, the GC-MS fingerprints of SS and SSC showed differences in their chromatographic profiling and relative contents (Figure 1). The circled peaks in section I and section III had higher contents in SS, whereas section II was relatively higher in SSC compared with 
in SS samples. This phenomenon reminded us that stir-fry processing could cause chemical profile changes in SS.

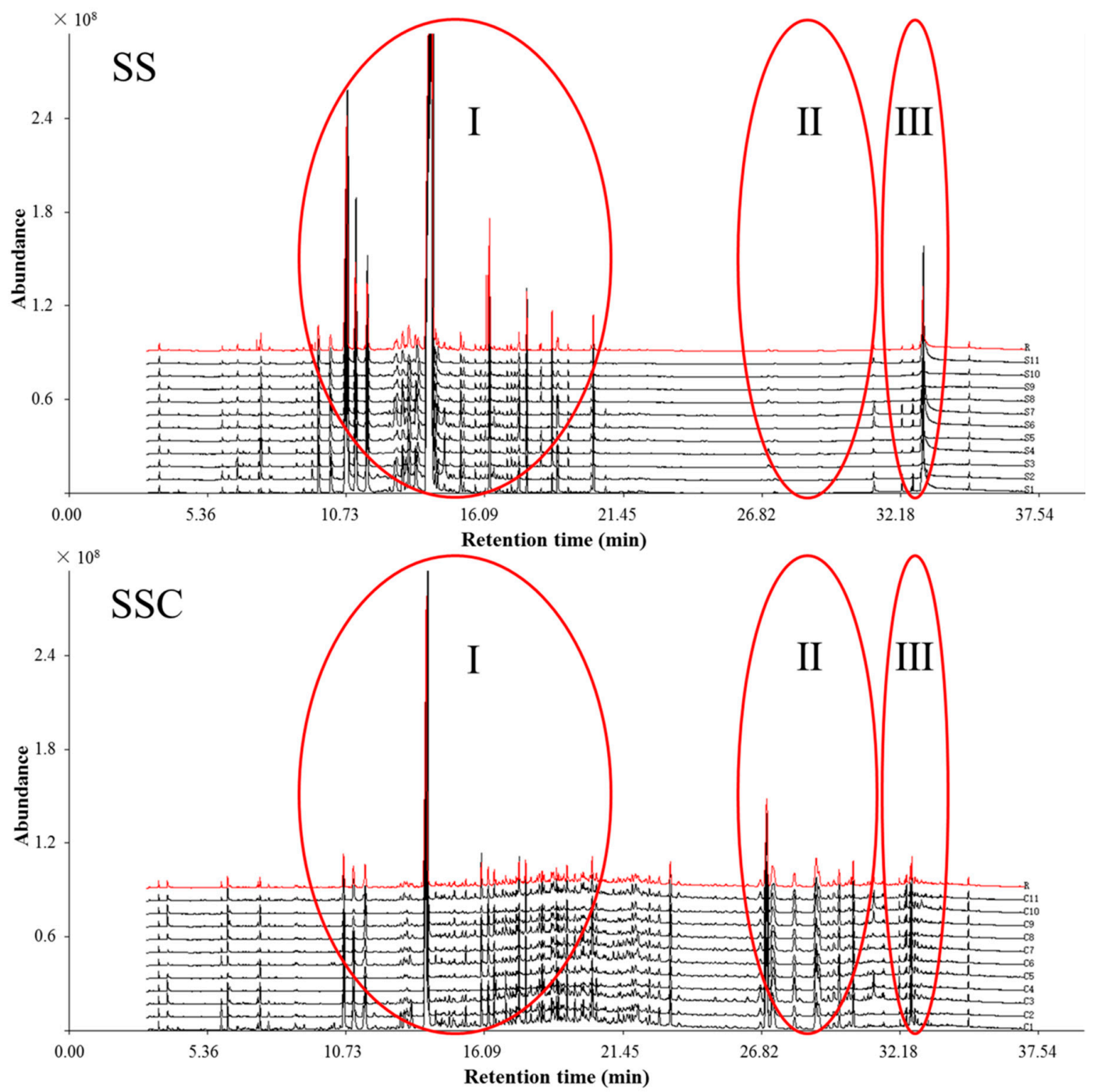

Figure 1. GC-MS fingerprints of Schizonepetae Spica (SS) and Schizonepetae Spica Carbonisata (SSC) samples and their reference chromatograms (R).

Table 1. Details of the 11 batches SS and SSC samples and their fingerprint similarities.

\begin{tabular}{cccccc}
\hline \multirow{2}{*}{ No. } & Voucher Specimen & Place of Collection & Date of Collection & \multicolumn{2}{c}{ Similarity } \\
\cline { 5 - 6 } & & & SS & SSC \\
\hline 1 & YP132581301 & Henan province & 29 November 2018 & 0.982 & 0.974 \\
2 & YP132581401 & Henan province & 17 January 2019 & 0.969 & 0.995 \\
3 & YP132581501 & Guangdong province & 8 January 2019 & 0.997 & 0.972 \\
4 & YP132581601 & Hubei province & 9 January 2019 & 0.995 & 0.962 \\
5 & YP132581701 & Hubei province & 10 January 2019 & 0.984 & 0.955 \\
6 & YP132581801 & Shanxi province & 9 January 2019 & 0.990 & 0.989 \\
7 & YP132580101 & Hebei province & 7 June 2018 & 0.989 & 0.978 \\
8 & YP132582301 & Hebei province & 9 January 2019 & 0.994 & 0.976 \\
9 & YP132582601 & Jiangsu province & 11 January 2019 & 0.991 & 0.995 \\
10 & YP132582901 & Jiangxi province & 11 January 2019 & 0.983 & 0.984 \\
11 & YP132583001 & Jiangxi province & 12 January 2019 & 0.994 & 0.977 \\
\hline
\end{tabular}




\subsubsection{Identification of Volatile Components from SS and SSC}

As shown in Table 2, the compositions of the volatile components of SS and SSC were analyzed. The number of identified peaks in SS and SSC were 39 and 62, accounting for $93.90 \%$ and $74.56 \%$ of their total volatile content, respectively. The main volatile constituents $(>1 \%)$ of SS included V18 (l-menthone, 13.05\%), V19 (menthofuran, 3.18\%), V20 (trans-5-methyl-2-(1-methylvinyl)cyclohexan-1-one, 2.84\%), V25 (2-allyl-4-methylphenol, 1.11\%), V26 (pulegone, 58.70\%), V37 (3-methyl-6-(1-methylethylidene)cyclohex-2-en-1-one, 3.21\%), V48 (caryophyllene, $1.28 \%$ ) and V83 (linolenic acid, 2.67\%), a result that was in accordance with the findings of recent studies [27,28]. The volatile composition and relative contents of SS notably changed after processing by stir-frying, as V18 (l-menthone, 1.84\%), V19 (menthofuran, 1.46\%), V20 (trans-5-methyl-2-(1-methylvinyl)cyclohexan-1-one, 1.75\%), V26 (pulegone, 27.44\%), V35 (2,5,6-trimethylbenzimidazole, 1.01\%), V61 (caryophyllene oxide, 1.40\%), V72 (3,7,11-trimethyl-1-dodecanol, 1.49\%), V73 (neophytadiene, 10.11\%), V75 (phytol acetate, 1.58\%), V77 (3,7,11,15-tetramethyl-2-hexadecen-1-ol, 3.95\%), V80 (methyl palmitate, 1.56\%) and V82 (methyl linolenate, $1.28 \%$ ) were determined to be the main volatile constituents of SSC. In addition, 22 compounds found in SS samples disappeared in SSC samples, while 45 compounds were newly generated and identified in SSC samples. However, in a previous study, it was found that the major compounds limonene and menthone detected in SH were obviously decreased in SHC, whereas the relative contents of another four components, isomenthone, isopulegone, pulegone and piperitone, were higher in SHC than in SH [21]. Different sample preparation methods, different medicinal parts of $\mathrm{SH}$ and different sources of $\mathrm{SH}$ specimens were most likely the reasons for the variance in the results. In our study, the differences among these variables may lead to efficacy differences in the SS and SSC samples.

\subsubsection{Multivariate Statistical Analyses}

To further investigate the change in chemical compositions between SS and SSC, the GC-MS data (84 identified peaks) were subjected to PCA analysis. The PCA $\left(R^{2} X=0.853, Q^{2}=0.788\right)$ score plot showed that the 22 samples were obviously separated from the two groups (Figure 2). The first two PCs explained $80.59 \%$ of the data variance (PC1 $=72.06 \%$ and PC2 $=8.53 \%$ ); of these, PC1 played a significant role in discriminating SS and SSC samples. SS was located on the negative side of PC1, while SSC was located on the positive side of PC1. The corresponding loading plot of PC1 (Figure 3) was used to find the components responsible for the separation between SS and SSC. The signals giving a positive effect in PC1 demonstrated that the corresponding ingredients were higher in SSC than in SS. In contrast, the signals with negative values indicated that the level of related components was higher in SS. The signals of major constituents V18 (l-menthone), V19 (menthofuran), V20 (trans-5-methyl-2-(1-methylvinyl)cyclohexan-1-one), V25 (2-allyl-4-methylphenol), V26 (pulegone), V37 (3-methyl-6-(1-methylethylidene)cyclohex-2-en-1-one), V48 (caryophyllene) and V83 (linolenic acid) gave a negative contribution to PC1. The signals with positive PC1 values included major components V35 (2,5,6-trimethylbenzimidazole), V61 (caryophyllene oxide), V72 (3,7,11-trimethyl-1-dodecanol), V73 (neophytadiene), V75 (phytol acetate), V77 (3,7,11,15-tetramethyl-2-hexadecen-1-ol), V80 (methyl palmitate) and V82 (methyl linolenate). The volatile components of SS have been recognized as the major constituents responsible for its biological effects. For example, pulegone, which is known for its pleasant odor, analgesia, and anti-inflammatory and antiviral properties [19,29], is a chemical indicator of SS in the Chinese Pharmacopoeia (2015 edition) [7]. L-Menthone also presents analgesia and antiviral effects [19]. Caryophyllene is a functional cannabinoid receptor type 2 agonist [30]. Menthofuran is widely used in flavorings and fragrances [31]. On the other hand, neophytadiene is a dominant metabolite in Urtica dioica L., a folk medicine that is commonly used as a hemostatic agent [32]. Therefore, these compounds might be the discriminant marker compounds when distinguishing SS from SSC, which are characterized by different medicinal properties. 
Table 2. Volatile compounds and their relative contents in SS and SSC.

\begin{tabular}{|c|c|c|c|c|c|c|c|}
\hline \multirow{2}{*}{ No. } & \multirow{2}{*}{ Compound } & \multirow{2}{*}{ Molecular Formula } & \multirow{2}{*}{ RI } & \multirow{2}{*}{ CAS } & \multirow{2}{*}{ Similarity Indices } & \multicolumn{2}{|c|}{ Relative Content (\%) } \\
\hline & & & & & & ss & SSC \\
\hline V1 & Butyl acetate & $\mathrm{C}_{6} \mathrm{H}_{12} \mathrm{O}_{2}$ & 818.86 & $123-86-4$ & $81.07 \%$ & & $0.24 \pm 0.06$ \\
\hline V2 & $(R)-(+)-3$-Methylcyclohexanone & $\mathrm{C}_{7} \mathrm{H}_{12} \mathrm{O}$ & 952.53 & $13368-65-5$ & $82.43 \%$ & $0.10 \pm 0.04$ & $0.23 \pm 0.16$ \\
\hline V3 & Benzaldehyde & $\mathrm{C}_{7} \mathrm{H}_{6} \mathrm{O}$ & 965.57 & $100-52-7$ & $93.42 \%$ & - & $0.52 \pm 0.10$ \\
\hline V4 & 1-Octen-3-ol & $\mathrm{C}_{8} \mathrm{H}_{16} \mathrm{O}$ & 983.39 & $3391-86-4$ & $78.46 \%$ & $0.18 \pm 0.06$ & \\
\hline V5 & (1R,2S,5S)-2-Methyl-5-(3-oxoprop-1-en-2-yl)cyclopentane-1-carbaldehyde & $\mathrm{C}_{10} \mathrm{H}_{14} \mathrm{O}_{2}$ & 993.51 & $5951-57-5$ & $85.63 \%$ & - & $0.07 \pm 0.02$ \\
\hline V6 & 1,3,8-p-Menthatriene & $\mathrm{C}_{10} \mathrm{H}_{14}$ & 1005.59 & $18368-95-1$ & $82.08 \%$ & $0.04 \pm 0.02$ & \\
\hline V7 & 2-Ethyl-1,4-dimethylbenzene & $\mathrm{C}_{10} \mathrm{H}_{14}$ & 1022.65 & $1758-88-9$ & $91.27 \%$ & & $0.15 \pm 0.03$ \\
\hline V8 & $E, E$-2,6-Dimethyl-1,3,5,7-octatetraene & $\mathrm{C}_{10} \mathrm{H}_{14}$ & 1023.77 & $460-01-5$ & $88.83 \%$ & $0.08 \pm 0.03$ & \\
\hline V9 & $D$-Limonene & $\mathrm{C}_{10} \mathrm{H}_{16}$ & 1026.40 & $5989-27-5$ & $95.44 \%$ & $0.38 \pm 0.35$ & $0.42 \pm 0.17$ \\
\hline V10 & 3,5-Dimethyl-2-cyclohexen-1-one & $\mathrm{C}_{8} \mathrm{H}_{12} \mathrm{O}$ & 1042.58 & $1123-09-7$ & $83.79 \%$ & - & $0.15 \pm 0.05$ \\
\hline V12 & 1-Ethenyl-3,5-dimethyl-benzene & $\mathrm{C}_{10} \mathrm{H}_{12}$ & 1088.07 & $5379-20-4$ & $88.24 \%$ & - & $0.15 \pm 0.04$ \\
\hline V13 & 2,5-Dimethylstyrene & $\mathrm{C}_{10} \mathrm{H}_{12}$ & 1089.74 & $2039-89-6$ & $80.65 \%$ & $0.07 \pm 0.03$ & - \\
\hline V14 & Linalool & $\mathrm{C}_{10} \mathrm{H}_{18} \mathrm{O}$ & 1100.42 & $78-70-6$ & $83.43 \%$ & $0.10 \pm 0.02$ & - \\
\hline V15 & trans-1-Methyl-4-(1-methylvinyl)cyclohex-2-en-1-ol & $\mathrm{C}_{10} \mathrm{H}_{16} \mathrm{O}$ & 1118.90 & $7212-40-0$ & $89.17 \%$ & $0.90 \pm 0.17$ & \\
\hline V16 & 1-Phenyl-1-butene & $\mathrm{C}_{10} \mathrm{H}_{12}$ & 1122.33 & $824-90-8$ & $83.12 \%$ & & $0.06 \pm 0.02$ \\
\hline V17 & cis-p-Mentha-2,8-dien-1-ol & $\mathrm{C}_{10} \mathrm{H}_{16} \mathrm{O}$ & 1133.26 & $3886-78-0$ & $91.38 \%$ & $0.81 \pm 0.12$ & \\
\hline V18 & l-Menthone & $\mathrm{C}_{10} \mathrm{H}_{18} \mathrm{O}$ & 1148.46 & $14073-97-3$ & $90.28 \%$ & $13.05 \pm 6.27$ & $1.84 \pm 1.75$ \\
\hline V19 & Menthofuran & $\mathrm{C}_{10} \mathrm{H}_{14} \mathrm{O}$ & 1159.04 & $494-90-6$ & $81.68 \%$ & $3.18 \pm 2.34$ & $1.46 \pm 1.11$ \\
\hline V20 & trans-5-Methyl-2-(1-methylvinyl)cyclohexan-1-one & $\mathrm{C}_{10} \mathrm{H}_{16} \mathrm{O}$ & 1171.24 & 29606-79-9 & $88.11 \%$ & $2.84 \pm 0.61$ & $1.75 \pm 0.56$ \\
\hline V21 & (-)-cis-Isopiperitenol & $\mathrm{C}_{10} \mathrm{H}_{16} \mathrm{O}$ & 1202.38 & $96555-02-1$ & $91.15 \%$ & $0.73 \pm 0.28$ & - \\
\hline V22 & 3-Methyl-2-(2-methyl-2-butenyl)-furan & $\mathrm{C}_{10} \mathrm{H}_{14} \mathrm{O}$ & 1208.98 & 15186-51-3 & $86.28 \%$ & - & $0.25 \pm 0.12$ \\
\hline $\mathrm{V} 23$ & 4,7-Dimethylbenzofuran & $\mathrm{C}_{10} \mathrm{H}_{10} \mathrm{O}$ & 1214.35 & $28715-26-6$ & $84.75 \%$ & - & $0.43 \pm 0.23$ \\
\hline V24 & trans-Pulegone oxide & $\mathrm{C}_{10} \mathrm{H}_{16} \mathrm{O}_{2}$ & 1218.24 & 13080-28-9 & $79.80 \%$ & - & $0.40 \pm 0.10$ \\
\hline V27 & Piperitone & $\mathrm{C}_{10} \mathrm{H}_{16} \mathrm{O}$ & 1258.51 & $89-81-6$ & $86.72 \%$ & $0.35 \pm 0.19$ & $0.22 \pm 0.14$ \\
\hline V28 & 5-Isopropenyl-2-methyl-2-cyclohexen-1-yl pivalate & $\mathrm{C}_{15} \mathrm{H}_{24} \mathrm{O}_{2}$ & 1276.52 & $1000124-59-2$ & $79.10 \%$ & - & $0.20 \pm 0.07$ \\
\hline V29 & 4-Ethyl-2-methoxyphenol & $\mathrm{C}_{9} \mathrm{H}_{12} \mathrm{O}_{2}$ & 1283.03 & $2785-89-9$ & $83.34 \%$ & & $0.36 \pm 0.14$ \\
\hline V30 & (7-Hydroxy-3,3-dimethyl-4-oxo-7-vinylbicyclo [3.2.0]hept-1-yl)acetaldehyde & $\mathrm{C}_{13} \mathrm{H}_{18} \mathrm{O}_{3}$ & 1290.48 & $1000156-78-3$ & $79.14 \%$ & $0.40 \pm 0.23$ & - \\
\hline V31 & (1S,3S,5S)-1-Isopropyl-4-methylenebicyclo[3 .1.0] hexan-3-yl acetate & $\mathrm{C}_{12} \mathrm{H}_{18} \mathrm{O}_{2}$ & 1294.44 & $139757-62-3$ & $83.38 \%$ & $0.20 \pm 0.03$ & - \\
\hline V32 & Thymol & $\mathrm{C}_{10} \mathrm{H}_{14} \mathrm{O}$ & 1297.47 & $89-83-8$ & $84.05 \%$ & & $0.30 \pm 0.15$ \\
\hline V33 & Carveol & $\mathrm{C}_{10} \mathrm{H}_{16} \mathrm{O}$ & 1312.88 & $99-48-9$ & $85.39 \%$ & $0.13 \pm 0.03$ & \\
\hline V34 & (4-Methoxymethoxy-hex-5-ynylidene)-cyclohexane & $\mathrm{C}_{14} \mathrm{H}_{22} \mathrm{O}_{2}$ & 1315.74 & $1000186-16-6$ & $80.43 \%$ & - & $0.15 \pm 0.08$ \\
\hline V35 & 2,5,6-Trimethylbenzimidazole & $\mathrm{C}_{10} \mathrm{H}_{12} \mathrm{~N}_{2}$ & 1328.07 & $3363-56-2$ & $88.88 \%$ & - & $1.01 \pm 0.61$ \\
\hline V36 & Carvyl acetate & $\mathrm{C}_{12} \mathrm{H}_{18} \mathrm{O}_{2}$ & 1335.28 & $97-42-7$ & $84.83 \%$ & $0.10 \pm 0.04$ & 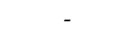 \\
\hline V37 & 3-Methyl-6-(1-methylethylidene)cyclohex-2-en-1-one & $\mathrm{C}_{10} \mathrm{H}_{14} \mathrm{O}$ & 1342.11 & $491-09-8$ & $90.88 \%$ & $3.21 \pm 0.55$ & $0.84 \pm 0.28$ \\
\hline V38 & 1,1,5-Trimethyl-1,2-dihydronaphthalene & $\mathrm{C}_{13} \mathrm{H}_{16}$ & 1354.07 & $1000357-25-8$ & $92.67 \%$ & - & $0.61 \pm 0.24$ \\
\hline V39 & 1,2,3,4-Tetrahydro-1,1,6-trimethylnaphthalene & $\mathrm{C}_{13} \mathrm{H}_{18}$ & 1357.25 & 475-03-6 & $83.46 \%$ & - & $0.17 \pm 0.10$ \\
\hline V40 & $\alpha$-Ethyl-4-methoxybenzenemethanol & $\mathrm{C}_{10} \mathrm{H}_{14} \mathrm{O}_{2}$ & 1371.02 & $5349-60-0$ & $87.85 \%$ & 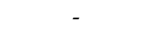 & $0.17 \pm 0.08$ \\
\hline V41 & $\alpha$-Copaene & $\mathrm{C}_{15} \mathrm{H}_{24}$ & 1376.65 & $1000360-33-0$ & $90.42 \%$ & $0.11 \pm 0.04$ & $0.27 \pm 0.19$ \\
\hline V42 & (-)- $\beta$-Bourbonene & $\mathrm{C}_{15} \mathrm{H}_{24}$ & 1385.02 & $5208-59-3$ & $92.16 \%$ & $0.13 \pm 0.03$ & $0.27=0.19$ \\
\hline V43 & Falcarinol & $\mathrm{C}_{17} \mathrm{H}_{24} \mathrm{O}$ & 1390.30 & $21852-80-2$ & $88.33 \%$ & - & $0.12 \pm 0.06$ \\
\hline V44 & $\beta$-Elemene & $\mathrm{C}_{15} \mathrm{H}_{24}$ & 1393.37 & 515-13-9 & $79.54 \%$ & $0.08 \pm 0.04$ & - \\
\hline
\end{tabular}


Table 2. Cont.

\begin{tabular}{|c|c|c|c|c|c|c|c|}
\hline \multirow{2}{*}{ No. } & \multirow{2}{*}{ Compound } & \multirow{2}{*}{ Molecular Formula } & \multirow{2}{*}{ RI } & \multirow{2}{*}{ CAS } & \multirow{2}{*}{ Similarity Indices } & \multicolumn{2}{|c|}{ Relative Content (\%) } \\
\hline & & & & & & ss & SSC \\
\hline V45 & Jasmone & $\mathrm{C}_{11} \mathrm{H}_{16} \mathrm{O}$ & 1399.03 & $488-10-8$ & $87.93 \%$ & $0.07 \pm 0.01$ & - \\
\hline V46 & 2-[(2Z)-2-Buten-1-yl]-4-hydroxy-3-methyl-2-cyclopenten-1-one & $\mathrm{C}_{10} \mathrm{H}_{14} \mathrm{O}_{2}$ & 1401.35 & $17190-74-8$ & $85.22 \%$ & $0.39 \pm 0.18$ & - \\
\hline V47 & 1-Methyl-4-[(2-methyl-3-butyn-2-yl)oxy]benzene & $\mathrm{C}_{12} \mathrm{H}_{14} \mathrm{O}$ & 1403.18 & 82719-54-8 & $87.66 \%$ & - & $0.90 \pm 0.50$ \\
\hline V48 & $\beta$-Caryophyllene & $\mathrm{C}_{15} \mathrm{H}_{24}$ & 1420.14 & $87-44-5$ & $94.76 \%$ & $1.28 \pm 0.78$ & $0.89 \pm 0.25$ \\
\hline V49 & $(3 \beta, 5 \alpha)-2$-Methylenecholestan-3-ol & $\mathrm{C}_{28} \mathrm{H}_{48} \mathrm{O}$ & 1455.41 & 22599-96-8 & $80.11 \%$ & & $0.60 \pm 0.17$ \\
\hline V50 & Humulene & $\mathrm{C}_{15} \mathrm{H}_{24}$ & 1458.11 & $6753-98-6$ & $86.35 \%$ & $0.15 \pm 0.10$ & \\
\hline V51 & $\beta$-Guaiene & $\mathrm{C}_{15} \mathrm{H}_{24}$ & 1479.26 & $88-84-6$ & $85.83 \%$ & - & $0.11 \pm 0.07$ \\
\hline V52 & 4-(2,4,4-Trimethyl-cyclohexa-1,5-dienyl)-but-3-en-2-one & $\mathrm{C}_{13} \mathrm{H}_{18} \mathrm{O}$ & 1484.88 & $1000187-51-9$ & $84.20 \%$ & - & $0.52 \pm 0.16$ \\
\hline V53 & Germacrene D & $\mathrm{C}_{15} \mathrm{H}_{24}$ & 1485.14 & 23986-74-5 & $90.97 \%$ & $0.80 \pm 0.56$ & - \\
\hline V54 & 1-Chlorooctadecane & $\mathrm{C}_{18} \mathrm{H}_{37} \mathrm{Cl}$ & 1496.85 & $3386-33-2$ & $84.04 \%$ & - & $0.61 \pm 0.17$ \\
\hline V56 & $(+)$ - $\delta$-Cadinene & $\mathrm{C}_{15} \mathrm{H}_{24}$ & 1523.45 & $483-76-1$ & $91.52 \%$ & $0.13 \pm 0.05$ & $0.96 \pm 0.30$ \\
\hline V57 & 4,5,9,10-Dehydroisolongifolene & $\mathrm{C}_{15} \mathrm{H}_{20}$ & 1542.96 & $156747-45-4$ & $88.25 \%$ & - & $0.51 \pm 0.25$ \\
\hline V58 & 3-(2-Methyl-propenyl)-1 $1 \mathrm{H}$-indene & $\mathrm{C}_{13} \mathrm{H}_{14}$ & 1559.02 & $1000187-78-5$ & $82.46 \%$ & - & $0.53 \pm 0.27$ \\
\hline V59 & 4,4-Dimethyl-3-(3-methylbut-3-enylidene)-2-methylenebicyclo[4.1.0]heptane & $\mathrm{C}_{15} \mathrm{H}_{22}$ & 1563.49 & 79718-83-5 & $82.83 \%$ & - & $0.49 \pm 0.28$ \\
\hline V60 & (-)-Spathulenol & $\mathrm{C}_{15} \mathrm{H}_{24} \mathrm{O}$ & 1579.60 & $77171-55-2$ & $81.56 \%$ & $0.14 \pm 0.04$ & $0.48 \pm 0.19$ \\
\hline V61 & Caryophyllene oxide & $\mathrm{C}_{15} \mathrm{H}_{24} \mathrm{O}$ & 1584.80 & $1139-30-6$ & $85.95 \%$ & $0.87 \pm 0.66$ & $1.40 \pm 0.66$ \\
\hline V62 & Geranyl isovalerate & $\mathrm{C}_{15} \mathrm{H}_{26} \mathrm{O}_{2}$ & 1595.20 & $109-20-6$ & $81.47 \%$ & & $0.56 \pm 0.17$ \\
\hline V63 & $(1 R, 3 E, 7 E, 11 R)-1,5,5,8$-Tetramethyl-12-oxabicyclo[9.1.0]dodeca-3,7-diene & $\mathrm{C}_{15} \mathrm{H}_{24} \mathrm{O}$ & 1612.71 & $19888-34-7$ & $83.83 \%$ & $0.06 \pm 0.05$ & \\
\hline V64 & (8S)-1-Methyl-4-isopropyl-7,8-dihydroxy-spiro[tricyclo[44.4.0.0(5,9)]-decane-10,2'-oxirane] & $\mathrm{C}_{15} \mathrm{H}_{24} \mathrm{O}_{3}$ & 1613.74 & 1000193-77-2 & $81.05 \%$ & 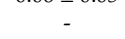 & $0.15 \pm 0.07$ \\
\hline V65 & $\alpha$-Santonin & $\mathrm{C}_{15} \mathrm{H}_{18} \mathrm{O}_{3}$ & 1628.24 & 481-06-1 & $81.28 \%$ & - & $0.19 \pm 0.16$ \\
\hline V66 & 10,10-Dimethyl-2,6-dimethylenebicyclo-[7.2.0]undecan-5 $\beta$-ol & $\mathrm{C}_{15} \mathrm{H}_{24} \mathrm{O}$ & 1637.26 & $19431-80-2$ & $84.47 \%$ & - & $0.29 \pm 0.11$ \\
\hline V67 & 2-[4-methyl-6-(2,6,6-trimethylcyclohex-1-enyl)hexa-1,3,5-trienyl]cyclohex-1-en-1-carboxaldehyde & $\mathrm{C}_{23} \mathrm{H}_{32} \mathrm{O}$ & 1646.83 & $1000216-09-2$ & $79.63 \%$ & - & $0.32 \pm 0.16$ \\
\hline V70 & $(1 R, 7 S, E)-7-$ Isopropyl-4,10-dimethylenecyclodec-5-enol & $\mathrm{C}_{15} \mathrm{H}_{24} \mathrm{O}$ & 1687.47 & 81968-62-9 & $81.29 \%$ & $0.03 \pm 0.02$ & $0.39 \pm 0.15$ \\
\hline V71 & $3,4^{\prime}$-Diethyl-1,1'-biphenyl & $\mathrm{C}_{16} \mathrm{H}_{18}$ & 1708.74 & 61141-66-0 & $88.07 \%$ & -0.00 & $0.45 \pm 0.09$ \\
\hline V72 & 3,7,11-Trimethyl-1-dodecanol & $\mathrm{C}_{15} \mathrm{H}_{32} \mathrm{O}$ & 1723.32 & 6750-34-1 & $80.42 \%$ & - & $1.49 \pm 0.38$ \\
\hline V73 & Neophytadiene & $\mathrm{C}_{20} \mathrm{H}_{38}$ & 1834.08 & $504-96-1$ & $90.35 \%$ & - & $10.11 \pm 3.20$ \\
\hline V74 & Fitone & $\mathrm{C}_{18} \mathrm{H}_{36} \mathrm{O}$ & 1842.16 & $502-69-2$ & $87.33 \%$ & $0.05 \pm 0.02$ & \\
\hline V75 & Phytol acetate & $\mathrm{C}_{22} \mathrm{H}_{42} \mathrm{O}_{2}$ & 1860.42 & $1000375-01-4$ & $85.59 \%$ & - & $1.58 \pm 0.60$ \\
\hline V76 & 2,4,7,14-Tetramethyl-4-vinyl-tricyclo[5.4.3.0(1,8)]tetradecan-6-ol & $\mathrm{C}_{20} \mathrm{H}_{34} \mathrm{O}$ & 1865.55 & 1000193-31-2 & $84.74 \%$ & - & $0.26 \pm 0.09$ \\
\hline V77 & 3,7,11,15-Tetramethyl-2-hexadecen-1-ol & $\mathrm{C}_{20} \mathrm{H}_{40} \mathrm{O}$ & 1879.81 & $102608-53-7$ & $85.05 \%$ & - & $3.95 \pm 1.53$ \\
\hline V78 & Hexadecanenitrile & $\mathrm{C}_{16} \mathrm{H}_{31} \mathrm{~N}$ & 1895.78 & $629-79-8$ & $81.46 \%$ & - & $0.63 \pm 0.19$ \\
\hline V79 & 1-Heptatriacotanol & $\mathrm{C}_{37} \mathrm{H}_{76} \mathrm{O}$ & 1914.84 & 105794-58-9 & $81.06 \%$ & - & $0.21 \pm 0.08$ \\
\hline V80 & Methyl palmitate & $\mathrm{C}_{17} \mathrm{H}_{34} \mathrm{O}_{2}$ & 1931.28 & $112-39-0$ & $88.36 \%$ & - & $1.56 \pm 0.48$ \\
\hline V81 & Methyl linoleate & $\mathrm{C}_{19} \mathrm{H}_{34} \mathrm{O}_{2}$ & 2091.82 & $112-63-0$ & $92.97 \%$ & 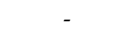 & $0.70 \pm 0.26$ \\
\hline V82 & Methyl linolenate & $\mathrm{C}_{19} \mathrm{H}_{32} \mathrm{O}_{2}$ & 2097.76 & $301-00-8$ & $90.66 \%$ & $0.08 \pm 0.06$ & $1.28 \pm 0.45$ \\
\hline V83 & Linolenic acid & $\mathrm{C}_{18} \mathrm{H}_{30} \mathrm{O}_{2}$ & 2149.50 & $463-40-1$ & $94.44 \%$ & $2.67 \pm 2.41$ & - \\
\hline V84 & 2,2'-Methylenebis(6-tert-butyl-4-methylphenol) & $\mathrm{C}_{23} \mathrm{H}_{32} \mathrm{O}_{2}$ & 2428.07 & $119-47-1$ & $87.78 \%$ & $0.11 \pm 0.02$ & $0.28 \pm 0.10$ \\
\hline
\end{tabular}

Relative content (\%) in the last two columns represents the mean \pm SD $(n=11$
the National Institute of Standards and Technology (NIST) MS database. 


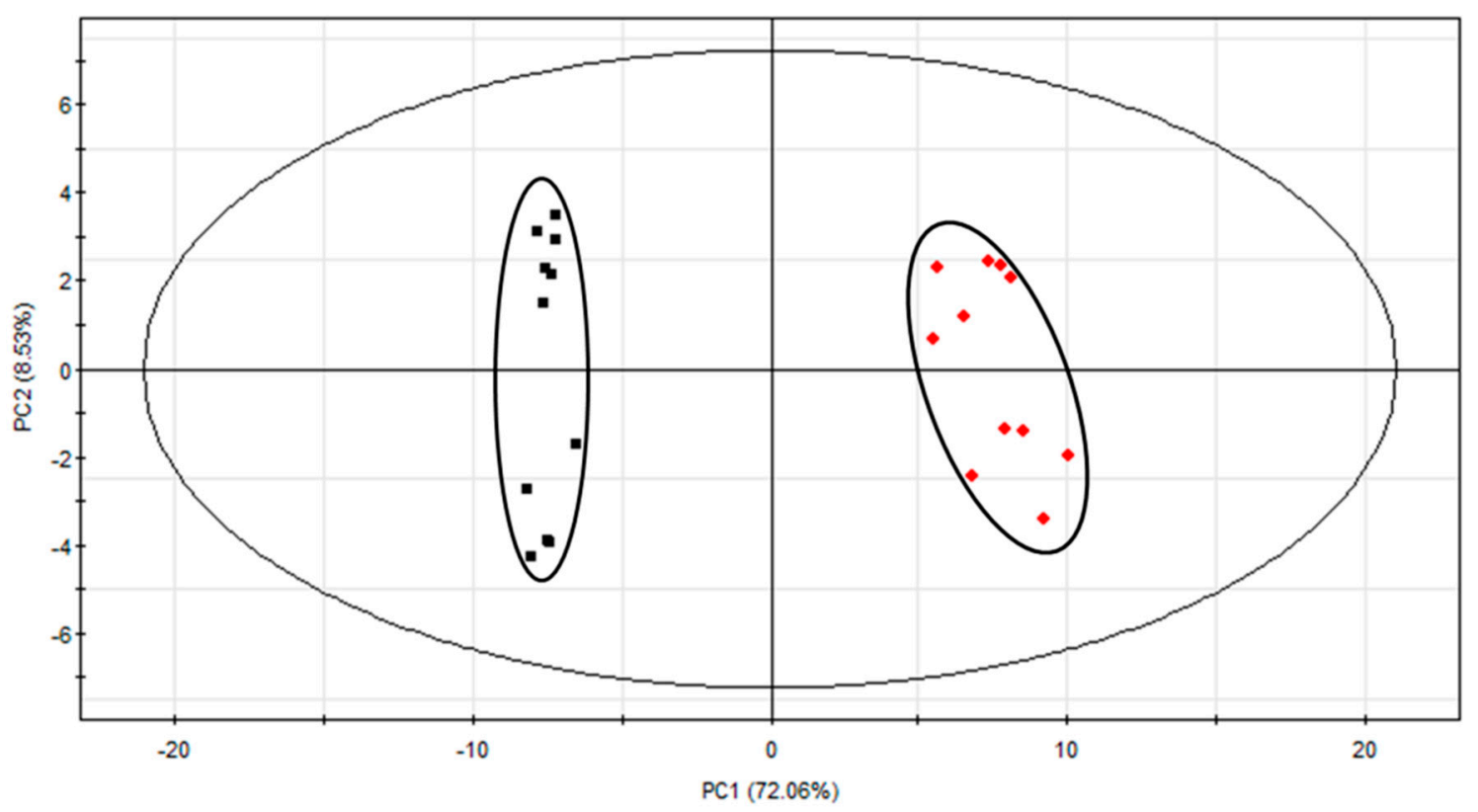

Figure 2. Principal component analysis (PCA) score plots based on gas chromatography-mass spectrometry (GC-MS) data of SS (black) and SSC (red).

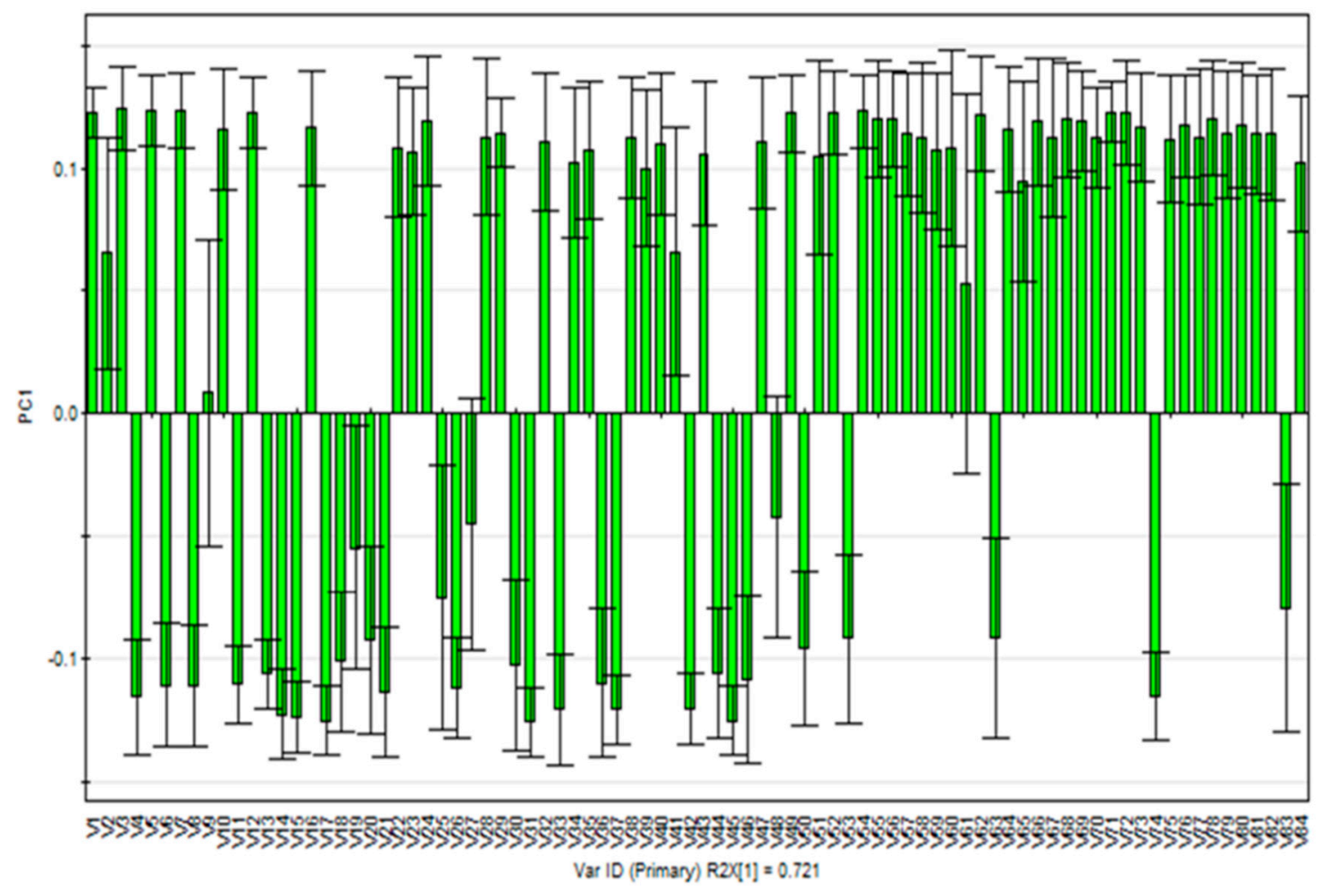

Figure 3. PC1 loading plot of the PCA results obtained from the GC-MS spectra.

2.1.4. Possible Mechanisms Involved in the Transformation of Volatile Compounds between SS and SSC

Building on the chemical marker analysis described above and the extensive organic synthesis knowledge accumulated by literature data [33-38], the possible mechanisms involved in the transformation of volatile compounds during stir-frying SS are demonstrated in Figure 4. For example, the significant decline in pulegone content in Table 2 revealed that the chemical reaction involved might generate an epoxidation reaction between the original pulegone (V26) and oxygen 
during stir-frying [33]. According to previous studies [19,29], pulegone has been demonstrated to have good biological activity in terms of anti-inflammatory and antiviral effects. However, evidence has proven that pulegone and its oxidative product $p$-cresol can cause severe hepatotoxicity [27]. Thus, the epoxidation reaction may occur in the process of preparing SSC by stir-frying raw SS, allowing SSC to contain less toxic pulegone. On the other hand, it has been found that $\beta$-Caryophyllene exhibits potent anti-inflammatory activity [39]. Stir-fry processing may also reduce the SS's anti-inflammatory property because of the changing of $\beta$-Caryophyllene (V48) to caryophyllene oxide (V61) and 10,10-dimethyl-2,6-dimethylenebicyclo-[7.2.0] undecan-5 $\beta$-ol (V66) [34,35]. Meanwhile, neophytadiene (V73) seemed to be generated from the dehydration reaction of 3,7,11,15-tetramethyl-2-hexadecen-1-ol (V77), a product that was generated from fitone (V74) by hydroxylation during stir-frying $[35,36]$. Neophytadiene is a major constituent in $U$. dioica, a folk medicine that is commonly used as a hemostatic agent [32]. Hence, the traditional use of SSC is in line with the observed increase in the amount of neophytadiene with plausible hemostatic activity. Likewise, linolenic acid (V83) was assumed to be transformed into methyl linolenate (V82) by esterification reaction during stir-frying [38]. Previous study has reported that linolenic acid shows bioactivities such as anti-inflammatory and antioxidant effects [40]. Therefore, it might be also related to the disappearance or significant decrease of linolenic acid (Table 2) and efficacy changing under the stir-fry processing of SS.

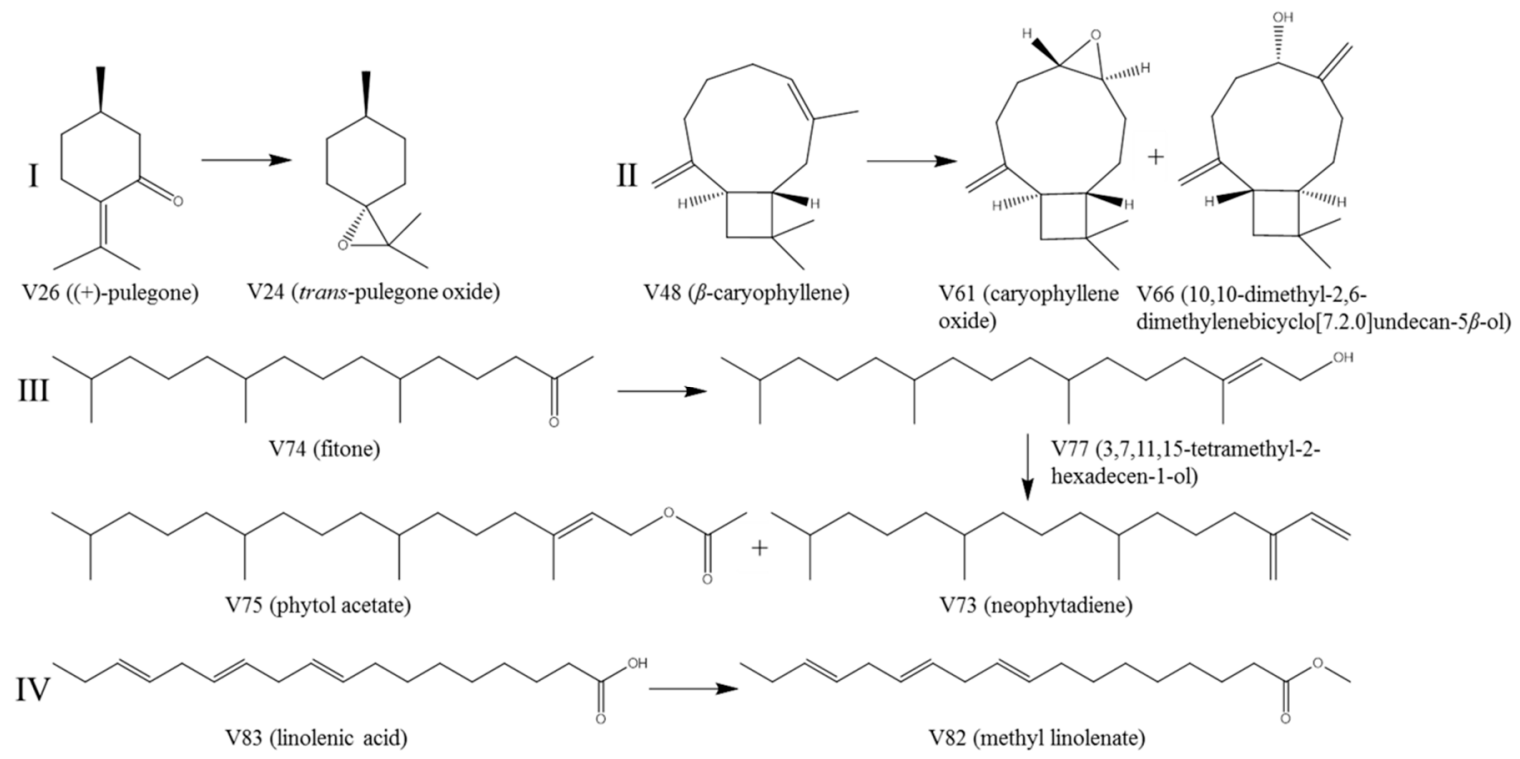

Figure 4. Possible mechanisms involved in the transformation of some main volatile components during stir-fry processing of SS.

\subsection{Comparison of the Nonvolatile Components of SS and SSC}

\subsubsection{Determination of Nonvolatile Compound Contents of SS and SSC}

Representative HPLC chromatograms of the SS and SSC samples are shown in Figure 5. The six nonvolatile compounds were identified using reference standards of luteolin-7-O- $\beta$-D-glucoside, apigenin-7-O- $\beta$-D-glucoside, hesperidin, rosmarinic acid, luteolin and apigenin, as well as comparing with literature [41-43]. Comparison of the chromatograms of SS and SSC revealed that stir-fry processing mainly caused quantitative changes among the nonvolatile compounds. The simultaneous determination of the six compounds in SS and SSC was analyzed (Table 3). Stir-fry processing significantly $(p<0.001)$ decreased the total nonvolatile compound contents of SS from $5.29 \pm 0.42 \mathrm{mg} / \mathrm{g}$ to $1.56 \pm 0.06 \mathrm{mg} / \mathrm{g}$. Compared with SS, the contents of four major ingredients (luteolin-7-O- $\beta$-D-glucoside, apigenin-7-O- $\beta$-D-glucoside, hesperidin and rosmarinic acid) decreased significantly $(p<0.001)$, whereas another two main components (luteolin and apigenin) increased significantly in SSC samples $(p<0.001)$. However, the intensity of some relatively polar compounds with shorter retention 
times on reversed-phase chromatography that were detected in SSC was markedly higher than those in SS (Figure 5). The identities of these compounds and the mechanisms involved in their transformation need to be further studied. Previous studies have demonstrated that stir-fry processing causes a significant increase in the total flavonoid and tannin contents of SH and SS [44-46]. Total flavonoids [47], tannins [48] and carbon dots [49] were proven to be active principles with hemostatic properties in SHC. In our research, the contents of total flavonoids (luteolin-7-O- $\beta$-D-glucoside, apigenin-7-O- $\beta$-D-glucoside, hesperidin, luteolin and apigenin) decreased remarkably after the SS samples were processed. A reasonable explanation for this result is that few flavonoids were detected in the ethanol extract of SSC.

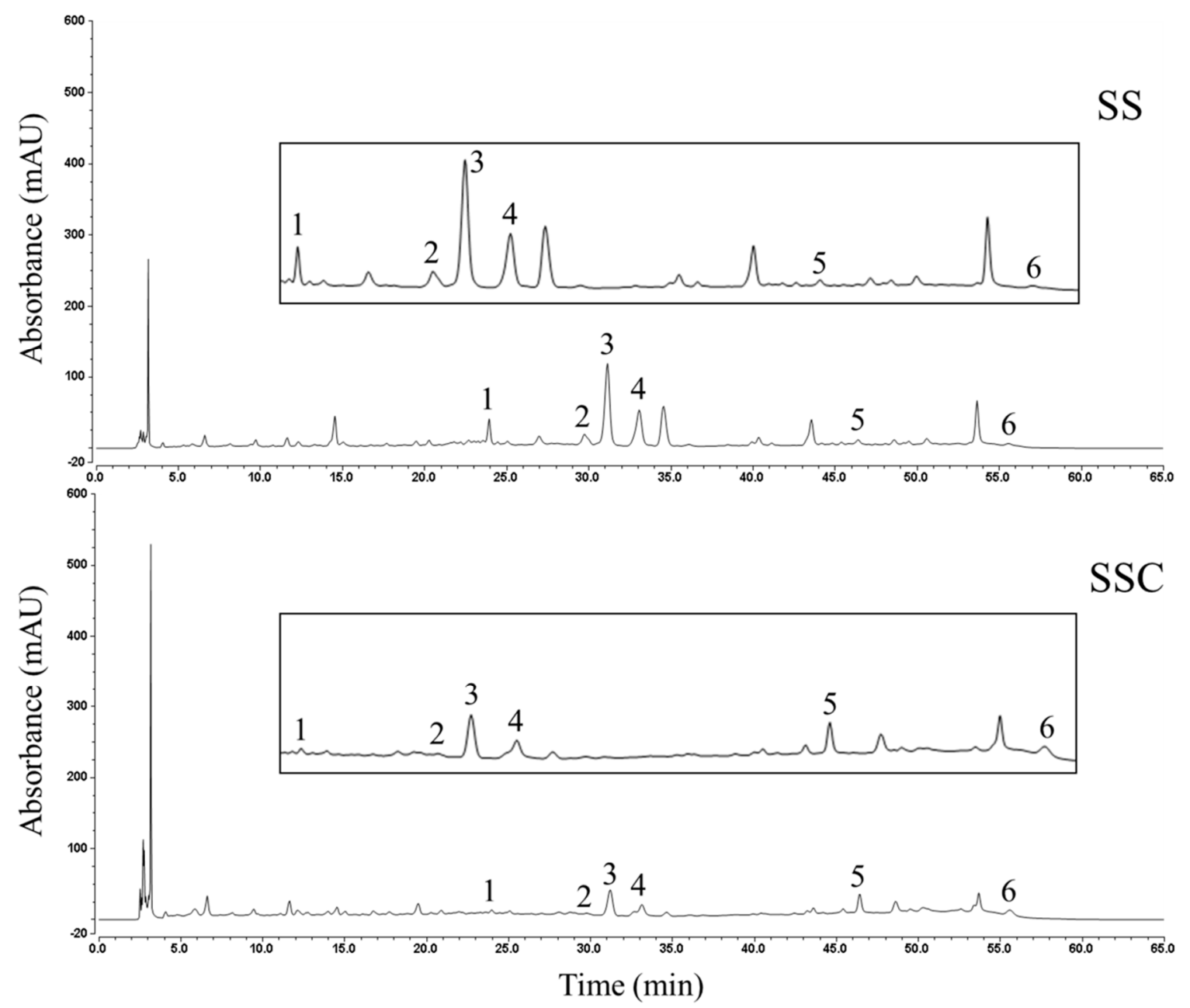

Figure 5. Representative HPLC chromatograms of SS and SSC samples. 1, luteolin-7-O- $\beta$-D-glucoside; 2 , apigenin-7-O- $\beta$-D-glucoside; 3 , hesperidin; 4 , rosmarinic acid; 5 , luteolin; and 6 , apigenin.

Table 3. Nonvolatile compound contents of SS and SSC.

\begin{tabular}{cccccccc}
\hline \multicolumn{7}{c}{ Contents of Nonvolatile Compounds (mg/g) } \\
\hline & $\begin{array}{c}\text { Luteolin-7-O- } \\
\beta \text {-D-glucoside }\end{array}$ & $\begin{array}{c}\text { Apigenin-7-O- } \\
\beta \text {-D-glucoside }\end{array}$ & Hesperidin & Rosmarinic Acid & Luteolin & \multirow{2}{*}{ Apigenin } & \multirow{2}{*}{ Total } \\
\hline SS & $0.40 \pm 0.06$ & $0.25 \pm 0.09$ & $2.97 \pm 0.99$ & $1.62 \pm 0.67$ & $0.04 \pm 0.04$ & $0.01 \pm 0.01$ & $5.29 \pm 0.42$ \\
SSC & $0.05 \pm 0.01^{* * *}$ & $0.02 \pm 0.01^{* * *}$ & $0.64 \pm 0.16^{* * *}$ & $0.40 \pm 0.11^{* * *}$ & $0.32 \pm 0.05^{* * *}$ & $0.13^{* * 0.03^{* * *}}$ & $1.56 \pm 0.06^{* * *}$ \\
\hline
\end{tabular}

Data are presented as the mean $\pm \mathrm{SD}(n=11)$. ${ }^{* * *} p<0.001$ vs. SS samples. The total nonvolatile compound contents were calculated as the sum of the contents of the 6 individual nonvolatile compounds. 
2.2.2. Possible Mechanisms Involved in the Transformation of the Nonvolatile Compounds between SS and SSC

In the present study, the changes in the contents of luteolin-7-O- $\beta$-D-glucoside and apigenin-7-O- $\beta$-D-glucoside probably contributed to the increases in luteolin and apigenin, respectively. The possible mechanisms involved in the transformation of these compounds are assumed in Figure 6. The results were consistent with previous reports in that glucoside could be hydrolyzed to aglycone under various conditions, including high temperature during stir-fry processing [43,50,51]. In addition, luteolin-7-O- $\beta$-D-glucoside has been documented to possess significant anti-inflammatory and antiviral effects [52,53]. Luteolin has been shown to be a potent hemostatic drug candidate [54]. Therefore, the increased contents of luteolin might result from the degradation of the glucosidic bond of luteolin-7-O- $\beta$-D-glucoside, which could be a main mechanism of stir-fry processing in SS.

I<smiles>CCOc1cc(-c2cc(=O)c3c(O)cc(OC4OC(CO)C(O)C(O)C4O)cc3o2)ccc1O</smiles>

II<smiles>O=c1cc(-c2ccc(O)cc2)oc2cc(OC3OC(CO)C(O)C(O)C3O)cc(O)c12</smiles>

Apigenin-7-O- $\beta$-D-glucoside

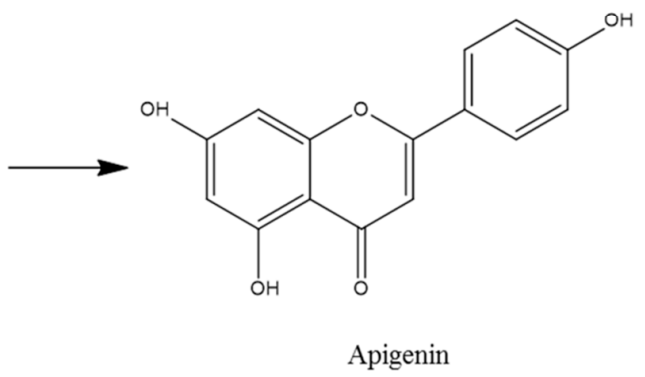

Figure 6. Possible mechanisms involved in the transformation of some main nonvolatile components during the stir-fry processing of SS.

\section{Materials and Methods}

\subsection{Materials and Reagents}

SS and SSC samples came from the same path (Table 1). SSC was processed in accordance with the Chinese Pharmacopoeia (2015 edition) [7]: dried SS slices (10-15 $\mathrm{mm}$ in length) were put in a pan and stir-fried until the surface became black and the interior became brown to avoid scorching. The identities of SS and SSC were confirmed by Dr. Ying Zhang, Jinan University, P. R. China. Voucher specimens were deposited at the Research Center for Traditional Chinese Medicine of Lingnan (Guangzhou, Southern China), Jinan University.

Ethyl acetate (analytical grade) was purchased from Aladdin (Aladdin, Shanghai, China). Methanol (HPLC grade), acetonitrile (HPLC grade) and formic acid (HPLC grade) were purchased from Fisher Scientific (Fair Lawn, NJ, USA). The chemical standards luteolin-7-O- $\beta$-D-glucoside, hesperidin, rosmarinic acid, luteolin and apigenin were purchased from Chengdu Ruifensi Biotechnology (Sichuan, China). apigenin-7-O- $\beta$-D-glucoside was purchased from Tianjun Biotechnology (Guangzhou, China). The purity of the compounds was greater than $95 \%$ as determined by HPLC. A mix of alkane analogues (GC grade, purity > 97\%), which was used as an internal quality standard for GC-MS analysis, was purchased from o2si (Charleston, SC, USA). Deionized water was obtained by passing distilled water through a Milli-Q purification system (Millipore, Bedford, MA, USA). All other reagents were of analytical grade. 


\subsection{Sample Preparation}

For GC-MS analysis, the steam distillation method was chosen according to the Chinese Pharmacopoeia (2015 edition) for the extraction of essential oils [7]. All samples were smashed and filtered through a 24-mesh sieve. The dried powder (50 g) was accurately weighed and transferred to a $1000 \mathrm{~mL}$ flask and soaked in $300 \mathrm{~mL}$ of redistilled water for $1 \mathrm{~h}$. Redistilled water was added from the top of the volatile oil determination apparatus until the water spilled into the flask, and $2 \mathrm{~mL}$ of ethyl acetate was added to the water layer. Then, the essential oils were extracted by water distillation for $4 \mathrm{~h}$. The volatile oil was separated from the water layer and leached into the ethyl acetate layer, and then the ethyl acetate layer was dried over anhydrous sodium sulfate for GC-MS analysis. The anhydrous essential oils were stored in dark glass vials at $-20^{\circ} \mathrm{C}$ until use.

For HPLC analysis, $2 \mathrm{~g}$ of the pulverized sample (filtered through a 24-mesh sieve) was weighed accurately and macerated in $20 \mathrm{~mL}$ of $75 \%$ ethanol. The sample was then extracted for $60 \mathrm{~min}$ by hot reflux extraction in a water bath. The supernatant of the extracts was filtered through a $0.45-\mu \mathrm{m}$ membrane before injection. The samples were stored in a refrigerator at $4{ }^{\circ} \mathrm{C}$ until use.

\subsection{Standard Solution Preparation}

A mixed standard stock solution containing the 6 reference compounds was prepared in methanol. Working standard solutions were prepared by diluting the mixed standard stock solution with methanol to give different concentrations within the following ranges for calibration curves: luteolin-7-O- $\beta$-D-glucoside, $0.796-159.2 \mu \mathrm{g} / \mathrm{mL}$; apigenin-7-O- $\beta$-D-glucoside, $0.48-48 \mu \mathrm{g} / \mathrm{mL}$; hesperidin, $2.084-625.2 \mu \mathrm{g} / \mathrm{mL}$; rosmarinic acid, $0.924-277.2 \mu \mathrm{g} / \mathrm{mL}$, luteolin, $0.472-141.6 \mu \mathrm{g} / \mathrm{mL}$ and apigenin, $0.4-120 \mu \mathrm{g} / \mathrm{mL}$. All standard solutions were filtered through a $0.45-\mu \mathrm{m}$ membrane before injection. The solutions were stored in a refrigerator at $4{ }^{\circ} \mathrm{C}$ before analysis.

\subsection{Apparatus and Chromatographic Conditions}

The GC-MS instrument used was an Agilent 7890B GC system coupled to an Agilent 7000C GC/MS Triple Quad mass spectrometer (Agilent, Santa Clara, CA, USA). Initial chromatographic separations of $1 \mu \mathrm{L}$ of sample were performed on a $15 \mathrm{~m} \times 250 \mu \mathrm{m}$ i.d. $\times 0.25 \mu \mathrm{m}$ film thickness HP-5 (Agilent) capillary column with a He flow rate of $1.0 \mathrm{~mL} / \mathrm{min}$ and an injection port temperature of $250{ }^{\circ} \mathrm{C}$ with a split ratio of $1: 10$. The oven temperature ramp was $3 \mathrm{~min}$ at $50{ }^{\circ} \mathrm{C}$, then $10^{\circ} \mathrm{C} / \mathrm{min}$ to $90^{\circ} \mathrm{C}$ where the temperature was held for $5 \mathrm{~min}$, then ramped at the rate of $10^{\circ} \mathrm{C} / \mathrm{min}$ to $160^{\circ} \mathrm{C}$ where the temperature was maintained for $10 \mathrm{~min}$, then a $20^{\circ} \mathrm{C} / \mathrm{min}$ ramp to $260^{\circ} \mathrm{C}$ where the temperature was held for $3 \mathrm{~min}$. The detector was operated at an ionization energy of $70 \mathrm{eV}$, and the $\mathrm{m} / \mathrm{z}$ values were recorded in the range 50-600 amu with a scan rate of $3.6 \mathrm{scan} / \mathrm{s}$ and a solvent delay of $3 \mathrm{~min}$. Components were identified using the National Institute of Standards and Technology (NIST) 2.2L Mass Spectra Database containing approximately 189,000 compounds, as well as comparing with literature [27,55-57].

HPLC analysis was performed on an UltiMate 3000 liquid chromatography system (Thermo Scientific, Bremen, Germany). Chromatographic separation was performed with an ACE Excel 5 C18 column (4.6 mm $\times 250 \mathrm{~mm}, 5 \mu \mathrm{m}$; ACE, Scotland, UK), and the column temperature was maintained at $30{ }^{\circ} \mathrm{C}$. The mobile phase was $0.1 \%$ formic acid aqueous solution (A) and acetonitrile (B) with a gradient program as follows: $0-20 \mathrm{~min}$, linear gradient from $8-20 \% \mathrm{~B} ; 20-30 \mathrm{~min}$, isocratic elution at $20 \% \mathrm{~B}$; $30-50 \mathrm{~min}$, linear gradient from $20-30 \% \mathrm{~B} ; 50-60 \mathrm{~min}$, linear gradient from 30-8\% B. The flow rate was $1 \mathrm{~mL} / \mathrm{min}$, and the injection volume was $10 \mu \mathrm{L}$. The detection wavelength was set at $283 \mathrm{~nm}$.

\subsection{Data Analysis}

The GC-MS fingerprint was performed by the professional software Similarity Evaluation System for Chromatographic Fingerprint of Traditional Chinese Medicine (Version 2004 A) composed by the Chinese Pharmacopoeia Committee. PCA was performed by SIMCA-P version 11.5 software (Umetrics, Umea, Sweden). Statistical significance was assessed by Student's two-tailed $t$-tests with GraphPad 
Prism v. 5.0 software (San Diego, CA, USA). Values of $p<0.05, p<0.01$ or $p<0.001$ were considered to be statistically significant.

\section{Conclusions}

This work reported, for the first time, the application of the combination of GC-MS and HPLC methods coupled with a strategy of corresponding data processing and statistical analysis to qualitatively and quantitatively distinguish SS and SSC and to identify marker compounds with significantly changed structures or contents during stir-fry processing. The GC-MS comparative analysis results of SS and SSC showed that 16 major constituents had a large contribution to the discrimination. HPLC analyses revealed that stir-fry processing remarkably increased the contents of two main ingredients but significantly reduced the contents of another four major constituents from SS. The change in the type and amount of these marker components is probably responsible for the different functions and pharmacological effects of SS and SSC as well as the observed hepatotoxicity. Finally, we speculated how some of these marker chemical changes occurred upon stir-fry processing. Hence, the potential mechanisms of stir-fry processing could be justified. The proposed strategy provided new clues for the investigation of the stir-frying-induced chemical transformation of SS and provided useful references for understanding the potential mechanisms of other processing methods.

Author Contributions: Conceptualization, H.C. and X.L.; Funding acquisition, H.C.; Investigation, X.L.; Formal analysis, X.L.; Resources, Y.Z.; Data curation, X.L.; Methodology, M.W. and Z.M.; Writing-original draft preparation, X.L.; Writing-review and editing, H.C., Y.Z., M.W. and Z.M. All authors have read and agreed to the published version of the manuscript.

Funding: This work was supported by the 6th National Academic Experience Inheritance Program of Famous Chinese Medicine Experts (Hui Cao) (No. 176-2017-XMZC-0166-01).

Conflicts of Interest: The authors declare no conflict of interest.

\section{Abbreviations}

$\begin{array}{ll}\text { SS } & \text { Schizonepetae Spica } \\ \text { SSC } & \text { Schizonepetae Spica Carbonisata } \\ \text { GC-MS } & \text { Gas chromatography-mass spectrometry } \\ \text { HPLC } & \text { High-performance liquid chromatography } \\ \text { CMM } & \text { Chinese medicinal material } \\ \text { TCM } & \text { Traditional Chinese medicine } \\ \text { SH } & \text { Schizonepetae Herba } \\ \text { SHC } & \text { Schizonepetae Herba Carbonisata } \\ \text { PCA } & \text { Principal component analysis }\end{array}$

\section{References}

1. Zhao, Z.Z.; Liang, Z.T.; Chan, K.; Lu, G.H.; Lee, E.L.M.; Chen, H.B.; Li, L. A unique issue in the standardization of Chinese materia medica: Processing. Planta Med. 2010, 76, 1975-1986. [CrossRef] [PubMed]

2. Lei, F. Lei Gong Pao Zhi Lun; Phoenix Science Press: Nanjing, China, 1985.

3. Su, T.; Zhang, W.W.; Zhang, Y.M.; Cheng, B.C.Y.; Fu, X.Q.; Li, T.; Guo, H.; Li, Y.X.; Zhu, P.L.; Cao, H.; et al. Standardization of the manufacturing procedure for Pinelliae Rhizoma Praeparatum cum Zingibere et Alumine. J. Ethnopharmacol. 2016, 193, 663-669. [CrossRef]

4. Yang, C.Y.; Guo, F.Q.; Zang, C.; Li, C.; Cao, H.; Zhang, B.X. The effect of ginger juice processing on the chemical profiles of Rhizoma Coptidis. Molecules 2018, 23, 380. [CrossRef] [PubMed]

5. Zhang, X.; Wang, Y.; Li, S.J.; Dai, Y.J.; Li, X.Q.; Wang, Q.H.; Wang, G.Y.; Ma, Y.L.; Gu, X.Z.; Zhang, C. The potential antipyretic mechanism of Gardeniae Fructus and its heat-processed products with plasma metabolomics using rats with yeast-induced fever. Front. Pharmacol. 2019, 10, 491. [CrossRef]

6. Zhang, X.; Wang, Y.; Li, X.Q.; Dai, Y.J.; Wang, Q.H.; Wang, G.Y.; Liu, D.P.; Gu, X.Z.; Yu, D.R.; Ma, Y.L.; et al. Treatment mechanism of Gardeniae Fructus and its carbonized product against ethanol-induced gastric lesions in rats. Front. Pharmacol. 2019, 10, 750. [CrossRef] 
7. State Commission of Chinese Pharmacopoeia. Pharmacopoeia of the People's Republic of China, 2015th ed.; China Medical Science Press: Beijing, China, 2015.

8. Wu, P. Shen Nong Ben Cao Jing; Sun, X.Y., Sun, F.J., Eds.; People's Medical Publishing House: Beijing, China, 1984.

9. Fung, D.; Lau, C.B.S. Schizonepeta tenuifolia: Chemistry, pharmacology, and clinical applications. J. Clin. Pharmacol. 2002, 42, 30-36. [CrossRef]

10. Shan, M.Q.; Qian, Y.; Yu, S.; Guo, S.C.; Zhang, L.; Ding, A.W.; Wu, Q.N. Anti-inflammatory effect of volatile oil from Schizonepeta tenuifolia on carrageenin-induced pleurisy in rats and its application to study of appropriate harvesting time coupled with multi-attribute comprehensive index method. J. Ethnopharmacol. 2016, 194, 580-586. [CrossRef]

11. Chen, S.G.; Cheng, M.L.; Chen, K.H.; Horng, J.T.; Liu, C.C.; Wang, S.M.; Sakurai, H.; Leu, Y.L.; Wang, S.D.; Ho, H.Y. Antiviral activities of Schizonepeta tenuifolia Briq. against enterovirus 71 in vitro and in vivo. Sci. Rep. 2017, 7, 1-15. [CrossRef]

12. Ng, Y.C.; Kim, Y.W.; Lee, J.S.; Lee, S.J.; Song, M.J. Antiviral activity of Schizonepeta tenuifolia Briquet against noroviruses via induction of antiviral interferons. J. Microbiol. 2018, 56, 683-689. [CrossRef]

13. Ding, A.W.; Kong, L.D.; Wu, H.; Wang, S.L.; Long, Q.J.; Yao, Z.; Chen, J. Research on hemostatic constituents in carbonized Schizonepeta tenuifolia Briq. China J. Chin. Mater. Med. 1993, 18, 535-538.

14. Ding, A.W.; Wu, H.; Kong, L.D.; Wang, S.L.; Gao, Z.Z.; Zhao, M.X.; Tan, M. Research on hemostatic mechanism of extracts from carbonized Schizonepeta tenuifolia Briq. China J. Chin. Mater. Med. 1993, 18, 598-600.

15. Zhang, Y. Application of jingjiesui in treating gynecological diseases. Clin. J. Chin. Med. 2016, 8, 69-70.

16. Du, C.Z.; Qin, J.P.; Chen, Y.P.; Cai, Y. GC-MS analysis of volatile oil components in Schizonepeta tenuifolia Briq. from various habitats. Hubei Agric. Sci. 2014, 53, 188-190.

17. Hu, J.; Shi, R.B.; Zhang, Y.H. Content determination of luteolin and hesperidin in the effective fractions of Spica Schizonepeta by HPLC. J. Beijing Univ. Tradit. Chin. Med. 2005, 28, 52-54.

18. Jiang, Y.H.; Jiang, D.Q.; Wu, K.J.; Peng, L.L. Contents determination of ursolic acid and oleanolic acid in different parts of Jiangxi Herba Schizonepetae and carbonized Herba Schizonepetae. China J. Tradit. Chin. Med. Pharm. 2016, 31, 1068-1070.

19. He, T.; Tang, Q.; Zeng, N.; Gong, X.P. Study on effect and mechanism of volatile oil of Schizonepetae Herba and its essential components against influenza virus. China J. Chin. Mater. Med. 2013, 38, 1772-1777.

20. Zhou, S.F.; Xue, C.C.; Yu, X.Q.; Wang, G. Metabolic activation of herbal and dietary constituents and its clinical and toxicological implications: An update. Curr. Drug Metab. 2007, 8, 526-553. [CrossRef]

21. Ye, D.J.; Ding, A.W.; Yu, L.; Cao, G.X.; Wu, M. Study on the composition of volatile oil in different medicinal parts of Schizonepetae Herba and its charred products. Bull. Chin. Mater. Med. 1985, 10, 19-21.

22. Zuo, M.L. Schizonepeta health products and processed products of different quality inspection. China Pharm. 2009, 18, 26-27.

23. Miao, X.Y. Pao Zhi Da Fa; Cao, H., Wu, M.H., Eds.; China Medical Science Press: Beijing, China, 2018.

24. Huang, Q.; Wu, D.L.; Wang, Y.; Ma, Y.L.; Yu, D.R.; Zhang, C. GC-MS analysis of volatile components in Scutellariae Radix before and after being charred. Chin. J. Exp. Tradit. Med. Form. 2016, 22, 9-12.

25. Zhang, L.; Shao, X.; Yu, S.; Bao, B.H.; Ding, A.W. HSGC fingerprint of volatile oil in Schizonepetae Spica. Chin. Tradit. Herb. Drugs 2012, 43, 1767-1769.

26. Wang, Q.; Wang, L.X.; Wang, X.Y.; Wang, Y.F. Chromatographic fingerprint for Schizonepeta tenuisfolia Briq. Chin. Tradit. Pat. Med. 2007, 29, 941-945.

27. Yu, S.; Chen, Y.W.; Zhang, L.; Shan, M.Q.; Tang, Y.P.; Ding, A.W. Quantitative comparative analysis of the bio-active and toxic constituents of leaves and spikes of Schizonepeta tenuifolia at different harvesting times. Int. J. Mol. Sci. 2011, 12, 6635-6644. [CrossRef]

28. Liu, X.; Zhang, Y.; Wu, M.; Ma, Z.; Cao, H. Color discrimination and gas chromatography-mass spectrometry fingerprint based on chemometrics analysis for the quality evaluation of Schizonepetae Spica. PLoS ONE 2020, 15, 1-15. [CrossRef]

29. Yamahara, J.; Matsuda, H.; Watanabe, H.; Sawada, T. Biologically active principles of crude drug, analgesic and anti-inflammatory effects of "Keigai (Schizonepeta tenuifolia Briq.)". Yakugaku Zasshi 1980, 100, 713-717. [CrossRef] 
30. Oliveira, G.L.D.; Machado, K.C.; Machado, K.C.; Da Silva, A.P.D.C.L.; Feitosa, C.M.; Almeida, F.R.D. Non-clinical toxicity of $\beta$-caryophyllene, a dietary cannabinoid: Absence of adverse effects in female Swiss mice. Regul. Toxicol. Pharm. 2018, 92, 338-346. [CrossRef]

31. Wang, Y.; Zhang, N. Review on the safety and fine processing technology of natural aromatic materials. Flavour Fragrance Cosmet. 2016, 4, 53-58.

32. Smoylovska, G.P. Identification of phytosterins in Urtica dioica L. (overground part). Zaporozhye Med. J. 2017, 1, 90-93. [CrossRef]

33. Riss, B.; Garreau, M.; Fricero, P.; Podsiadly, P.; Berton, N.; Buchter, S. Total synthesis of TMS-ent-bisabolangelone. Tetrahedron 2017, 73, 3202-3212. [CrossRef]

34. Uchida, T.; Matsubara, Y.; Koyama, Y. Structures of two novel sesquiterpenoids formed by the lead tetraacetate oxidation of $\beta$-caryophyllene. Agric. Biol. Chem. 1989, 53, 3011-3015.

35. Yamazaki, S. An effective procedure for the synthesis of acid-sensitive epoxides: Use of 1-methylimidazole as the additive on methyltrioxorhenium-catalyzed epoxidation of alkenes with hydrogen peroxide. Org. Biomol. Chem. 2010, 8, 2377-2385. [CrossRef]

36. Duhamel, L.; Ancel, J.E. Utilization of functional vinylic organometallics for convergent syntheses of phytol from 6-methylhept-5-en-2-one. J. Chem. Res. 1990, 5, 154-155.

37. Grossi, V.; Rontani, J.F. Photosensitized oxygenation of phytadienes. Tetrahedron Lett. 1995, 36, 3141-3144. [CrossRef]

38. Damm, M.; Kappe, C.O. High-throughput experimentation platform: Parallel microwave chemistry in HPLC/GC vials. J. Comb. Chem. 2009, 11, 460-468. [CrossRef] [PubMed]

39. Li, H.; Wang, D.F.; Chen, Y.J.; Yang, M.S. $\beta$-Caryophyllene inhibits high glucose-induced oxidative stress, inflammation and extracellular matrix accumulation in mesangial cells. Int. Immunopharmacol. 2020, 84, 1-9. [CrossRef]

40. Ok, F.; Kaplan, H.M.; Kizilgok, B.; Demir, E. Protective effect of $\alpha$-linolenic acid on lipopolysaccharide-induced orchitis in mice. Andrologia 2020, 00,1-6. [CrossRef]

41. Fan, J.X.; Wang, S.; Meng, X.S.; Bao, Y.R.; Li, T.J. Determination of six flavonoids in Schizonepeta tenuifolia from different areas by HPLC. Chin. Tradit. Herb. Drugs 2017, 48, 2292-2295.

42. Hu, J.H.; Liu, L.L.; Zhang, Y.J.; Xiao, W. Determination of cafferic acid and rosmarinic acid in Perilla frutescens leaves and Schizonepata tenuifolia by HPLC. Chin. Tradit. Herb. Drugs 2015, 46, 2155-2159.

43. Bolzon, L.B.; Dos Santos, J.S.; Silva, D.B.; Crevelin, E.J.; Moraes, L.A.B.; Lopes, N.P.; Assis, M.D. Apigenin-7-O-glucoside oxidation catalyzed by P450-bioinspired systems. J. Inorg. Biochem. 2017, 170, 117-124. [CrossRef]

44. Bao, B.H.; Yang, J.P.; Zhang, L.; Ding, A.W. Quantitative analysis of flavonoid in Schizonepetae Spica. J. Nanjing Univ. Tradit. Chin. Med. 2004, 20, 124-125.

45. Bao, B.H.; Zhang, L.; Ding, A.W. Quantitative analysis of flavonoid in Schizonepeta tenuifolia Briq. Lishizhen Med. Mater. Med. Res. 2004, 15, 264-265.

46. Dong, C.H.; Liu, B.; Zhu, X. Study best processing degree of carbonized catnip. Qilu Pharm. Aff. 2006, 25, 560-599.

47. Zhang, L.; Bao, B.H.; Sun, L.; Ding, A.W. Optimum processing technique of Herba Schizonepetae Carbonisatus by orthogonal design. Chin. Tradit. Herb. Drugs 2005, 36, 370-372.

48. Cao, L.L.; Li, X.; Zhang, L. Experimental study on the effect of Schizonepetae Spica Carbonisata and its effective fractions on rat coagulation system. Chin. Tradit. Pat. Med. 2010, 32, 611-613.

49. Zhang, M.L.; Zhao, Y.; Cheng, J.J.; Liu, X.M.; Wang, Y.Z.; Yan, X.; Zhang, Y.; Lu, F.; Wang, Q.G.; Qu, H.H. Novel carbon dots derived from Schizonepetae Herba Carbonisata and investigation of their haemostatic efficacy. Artif. Cell Nanomed. B 2018, 46, 1562-1571. [CrossRef]

50. Kurkin, V.A.; Kharisova, A.V. Flavonoids of Carthamus tinctorius flowers. Chem. Nat. Compd. 2014, 50, 446-448. [CrossRef]

51. Li, S.L.; Yan, R.; Tam, Y.K.; Lim, G. Post-harvest alteration of the main chemical ingredients in Ligusticum chuanxiong hort. (Rhizoma chuanxiong). Chem. Pharm. Bull. 2007, 55, 140-144. [CrossRef]

52. Ma, S.C.; Liu, Y.; Paul, B.P.H.; Yang, Y.; Vincent, O.E.C.; Spencer, H.S.L.; Song, F.L.; Lu, J.; Lin, R.C. RP-HPLC determination of hederagenin and oleanolic acid in Flos Lonicerae Japonicae. Chin. J. Pharm. Anal. 2006, 26, 885-887. 
53. Ma, S.C.; Liu, Y.; Paul, B.P.H.; Yang, Y.; Vincent, O.E.C.; Spencer, H.S.L.; Song, F.L.; Lu, J.; Lin, R.C. Antiviral activities of flavonoids isolated from Lonicera japonica Thunb. Chin. J. Pharm. Anal. 2006, 26, 426-430.

54. Lin, Z.C.; Fang, Y.J.; Huang, A.Y.; Chen, L.Y.; Guo, S.H.; Chen, J.W. Chemical constituents from Sedum aizoon and their hemostatic activity. Pharm. Biol. 2014, 52, 1429-1434. [CrossRef]

55. Chun, M.H.; Kim, E.K.; Lee, K.R.; Jung, J.H.; Hong, J. Quality control of Schizonepeta tenuifolia Briq by solid-phase microextraction gas chromatography/mass spectrometry and principal component analysis. Microchem. J. 2010, 95, 25-31. [CrossRef]

56. Liu, C.C.; Srividya, N.; Parrish, A.N.; Yue, W.; Shan, M.Q.; Wu, Q.N.; Lange, B.M. Morphology of glandular trichomes of Japanese catnip (Schizonepeta tenuifolia Briquet) and developmental dynamics of their secretory activity. Phytochemistry 2018, 150, 23-30. [CrossRef]

57. Duan, S.L.; Zeng, W.X.; Sun, L.L. Chemical constituents of volatile oils in drug pair of menthae Haplocalycis herba and Schizonepetae spica by GC-MS. Chin. J. Exp. Tradit. Med. Form. 2015, 21, 50-54.

Sample Availability: Samples of the compounds are available from the authors.

(C) 2020 by the authors. Licensee MDPI, Basel, Switzerland. This article is an open access article distributed under the terms and conditions of the Creative Commons Attribution (CC BY) license (http://creativecommons.org/licenses/by/4.0/). 